\title{
ReMed: Jahresbericht 2019
}

ReMed, Leitungsausschuss

\author{
ReMed ist das Unterstützungsnetzwerk, welches Sie als Ärztin und Arzt in Krisen \\ unterstützt und für die Förderung und Prävention der eigenen Gesundheit sensi- \\ bilisiert. Innerhalb von 72 Stunden steht Ihnen ReMed unterstützend zur Seite. \\ Der Kontakt zu ReMed kann auch über Personen aus dem Umfeld der betroffenen \\ Ärztinnen und Ärzte erfolgen. Die Erhaltung der Gesundheit und ärztlichen Funk- \\ tionalität sowie die Gewährleistung der Patientensicherheit und der hohen Quali- \\ tät in der medizinischen Versorgung stehen dabei an erster Stelle.
}

\section{Auf die Ärzteschaft ausgerichtet - für Ärzte von Ärzten}

ReMed wird durch die Verbindung der Schweizer Ärztinnen und Ärzte FMH getragen. Die operative Umsetzung des Unterstützungsnetzwerks findet ausserhalb der FMH-Strukturen statt. ReMed orientiert sich in seiner Vorgehensweise an den gesetzlichen Rahmenbedingungen sowie an der Standesordnung der FMH.

Das ReMed Angebot beruht auf Vertraulichkeit und Selbstverantwortung der einzelnen Ärztin, des einzelnen Arztes. ReMed handelt immer und nur mit dem ausdrücklichen Einverständnis der ratsuchenden Ärztin, des ratsuchenden Arztes. Diese geniessen ab Kontaktaufnahme innerhalb von ReMed die gleichen Rechte wie andere Patienten: Es gilt das Patientengeheimnis und die Datenschutzbestimmungen werden jederzeit eingehalten. ReMed ist kein Aufsichtsorgan und hat weder Abklärungs- noch Sanktionskompetenzen.

Auch wenn ReMed von der FMH finanziert wird, hat die Standesorganisation zu keinem Zeitpunkt Einblick in die Personen- und Betreuungsakten. ReMed ist an das ärztliche Berufsgeheimnis gebunden, ärztliche und administrative Aufgaben sind klar getrennt.

\section{Vertraulichkeit und Selbstverantwortung}

Wenn Sie sich an ReMed wenden, gehen Sie keine Verpflichtungen ein - nichts geschieht ohne Ihre Zustimmung. Und was Sie ReMed anvertrauen, bleibt vertraulich: Das Unterstützungsnetzwerk ist an das Patientengeheimnis gebunden.

Die ratsuchende Ärztin, der ratsuchende Arzt geniesst bei den bei ReMed mitarbeitenden Ärzten und ihren Hilfspersonen in Praxis und Spital ab Kontaktaufnahme die gleichen Rechte wie andere Patienten: es gilt das Patientengeheimnis und die Datenschutzbestimmungen werden eingehalten. Es gelten zudem dieselben gesetzlichen Ausnahmen vom ärztlichen Berufsgeheimnis. Grundsätzlich haben nur direkt in einen Fall involvierte Personen von ReMed Zugriff zu den persönlichen Daten. Auch Ratsuchende haben ein Einsichtsrecht in die über sie angelegten Daten gemäss den Datenschutzbestimmungen.

\section{Effektive Kommunikation}

Durch effektive Kommunikation mittels diverser Artikel in der Tagespresse, in medizinischen Fachzeitschriften, mit Radiobeiträgen sowie mit Testimonials in der Schweizerischen Ärztezeitung (in welchen Betroffene von ihrer Krise und ihren Erfahrungen mit ReMed berichteten) als auch mit Präsentationen in Spitälern, Kliniken, Instituten, lokalen Ärztegesellschaften, Kongressen wurde im vergangenen Jahr auf das breite Angebot von ReMed aufmerksam gemacht und die Zielgruppen erreicht. Auf internationaler Ebene, am EAPH Kongress in Oslo, durften die ReMed-Dienstleistungen sowie Ergebnisse aus der externen Evaluation präsentiert werden, die auf grosses Interesse stiessen.

\section{Kontaktaufnahmen im Jahr 2019}

Die Kontaktaufnahmen zu ReMed sind im vergangenen Jahr um 10\% auf 162 Fälle angestiegen. Allein im Februar 2019 wurde das Netzwerk 20 Mal angerufen. Die andauernde Zunahme beweist die Dringlichkeit der ReMed-Dienstleistungen deutlich. Bei den Kontaktaufnahmen, welche von den Erstberatenden betreut wurden, erfolgten 110 Anfragen aus der Deutschschweiz, 50 Fälle aus der Romandie und 2 Fälle aus dem Tessin. Die Kontaktsuchenden waren zu 60\% weiblich und zu 40\% männlich. Die Mehrheit der Kon- 
Gründe für Kontaktaufnahmen (Mehrfachnennungen möglich)

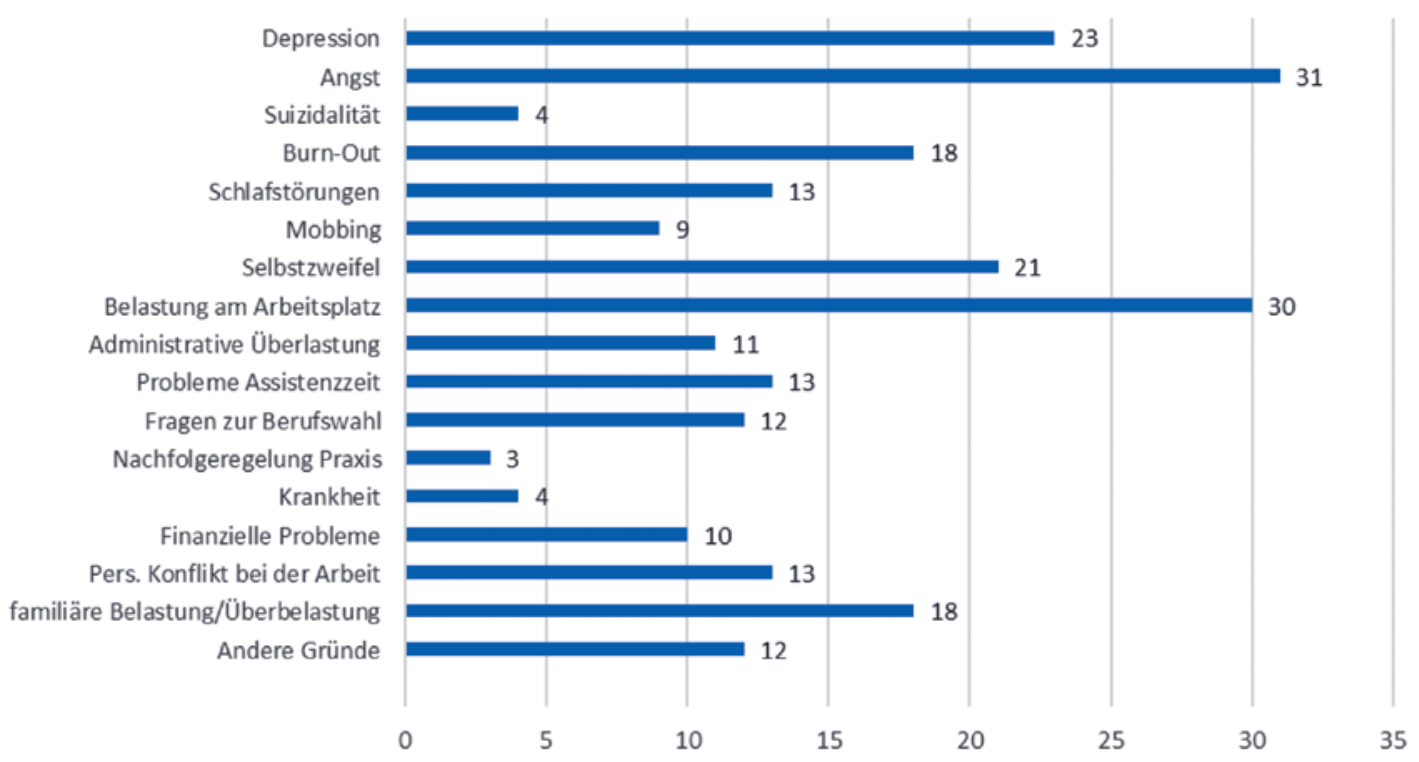

Abbildung 1: Gründe für Kontaktaufnahme bei ReMed (Mehrfachnennungen möglich).

taktsuchenden (53\%) kam aus dem stationären Bereich. Die Problematik «Angst» (u.a. Zukunftsängste, Laufbahnentscheide, vor Praxisab- und Übergabe, vor Behandlungsfehlern) gefolgt von den Problemen «Belastung am Arbeitsplatz» und «Depression» waren die Hauptgründe der Kontaktaufnahme (vgl. Abb. 1).

\section{Ärztegesundheit als Voraussetzung für Qualität in der Medizin}

An der Berner Fachhochschule wird ab 2020 ein CAS «Qualität in der Medizin für patientennahe Arbeitspraxis» angeboten. ReMed wird im Modul betr. Ärztegesundheit mit eingebunden und mithelfen, ein umfassendes Verständnis zur Gesundheitsförderung und Prävention zu schaffen.

\section{Coach my Career Mentoring Programm}

Nicht nur am Ende des Studiums, sondern auch danach stehen junge Ärztinnen und Ärzte vor Entscheidungen, die ihr ganzes Berufsleben beeinflussen: Bewege ich mich in Richtung Forschung oder Industrie? Eröffne ich eine Praxis oder beginne ich eine Karriere im Spital oder an der Universität? Für Rat bei solchen Fragen scheint oft niemand da zu sein. Deshalb haben FMH, VLSS, VSAO, mfe und SWIMSA - mit Unterstützung des SIWF - das generationenübergreifende Mentoring-Programm Coach my Career lanciert. Coach my Career versteht sich als Ergänzung zu Angeboten von Fachgesellschaften, Universitäten oder Spitälern und auch zu ReMed. Entsprechend fand ein spannender und konstruktiver Austausch zwischen ReMed und Coach my Career statt. So kann sichergestellt werden, dass die beiden Programme sich ergänzen und gegenseitig unterstützen. Mehr Informationen finden Sie online unter: $\mathrm{fmh}$.ch/dienstleistungen/stationaere-tarife/coach-my-career.cfm

\section{Trauriger Verlust für das Netzwerk}

Leider mussten wir im vergangenen Jahr Abschied nehmen von Dr. med. Peter Birchler, einem Gründungsmitglied und aktivem Mitglied des Leitungsausschusses von ReMed. Wir verlieren mit Peter Birchler einen Schöpfer des Netzwerks, welcher ReMed mit Scharfsinn und Weitblick belebt und mit höchstem Engagement gestaltet und geprägt hat. Wir werden ihn und seinen unermüdlichen Einsatz in bester Erinnerung behalten.

\section{Erfahrungsberichte und Dokumentationen}

Besuchen Sie die Homepage www.swiss-remed.ch und finden Sie interessante Erfahrungsberichte und Dokumentationen sowie alle wertvollen Informationen über das Unterstützungsnetzwerk.

Das nationale Programm ReMed mit dem Leitungsausschuss und den Netzwerkmitgliedern wird auch im Jahr 2020 für Ärztinnen und Ärzte in Krisensituationen da sein. Die betroffenen Ärztinnen und Ärzten können jederzeit über die 24-Stunden-Hotline (0800 0 73633) oder per remed[at]hin.ch Beratung und Hilfe anfordern. 